\title{
A Combinatorial Geometrical Approach to the Error Performance of Multidimensional Finite Lattice Constellations
}

\author{
Koralia N. Pappi, Nestor D. Chatzidiamantis, and George K. Karagiannidis \\ Department of Electrical \& Computer Engineering, Aristotle University of Thessaloniki, Thessaloniki, Greece, \\ E-mails: \{kpappi, nestoras, geokarag\}@auth.gr
}

\begin{abstract}
We present a novel combinatorial geometrical approach for evaluating the performance of multidimensional finite lattice constellations in Additive White Gaussian Noise (AWGN). More specifically, we present an analytical expression for the exact symbol error probability (SEP) of multidimensional signal sets, which is then used to derive a tight closed-form lower bound of the SEP, named Multiple Sphere Lower Bound (MLSB). Simulations are used to compare the proposed bound with the performance of various lattice constellations. It is clearly demonstrated that the MSLB can be efficiently used in finite lattice constellations, with arbitrary structure, dimension and rank. Furthermore, it can be easily extended to the case of fading channels, where multidimensional signal sets are commonly used to combat fading degradation.
\end{abstract}

\section{INTRODUCTION}

The employment of Signal Space Diversity (SSD) -a method which has been introduced in [1] to compensate for the degradation caused by fading channels- to multidimensional lattice constellations, has attracted the interest of both academia and industry. By rotating the multidimensional signal constellations and performing component interleaving, new signal sets can be designed to achieve diversity gain without any additional requirements for power, frequency or multiple antennas. Such signal sets that have the potential to achieve full diversity, have been presented in the pioneer works [2]-[5] and have been carved from rotated lattices which meet the criterion of the maximization of the minimum product distance.

Although the evaluation of the performance of such rotated multidimensional signal sets can be an important tool in their design, the study of their exact symbol error probability (SEP) is in general a hard problem and various methods have been presented to face it, based on either approximations [6] or union bounds [7]. Only recently, some exact expressions for the SEP of two-dimensional constellations in the presence of Rice fading have been presented in [8]; however, the extension of such analysis to multiple dimensions seems to be complicated.

The sphere lower bound (SLB), which dates back to Shannon's work [9], has been proposed as an efficient tool for evaluating the performance of multidimensional constellations [10]-[12]. In [13], the SLB, which approximates the Voronoi cell with a sphere of the same volume, was evaluated for Nakagami- $m$ block fading channels. It was clearly demon- strated that, although it always acts as a lower bound for infinite lattices, it is not generally a lower bound for finite lattice constellations. To the best of the authors' knowledge, a lower bound for these constellations has not been previously presented.

In this paper, we provide an analytical framework for the SEP evaluation of multidimensional finite lattice constellations. Our analysis can be efficiently applied in multidimensional signal sets, with arbitrary structure, dimension and rank, taking into account their geometrical characteristics. More specifically, we introduce a combinatorial geometrical approach for the error performance evaluation of these constellations, based on the properties of multidimensional lattice parallelotopes. Following this approach, we derive an analytical expression for the SEP of multidimensional finite lattice constellations, which is then lower-bounded by a novel closed-form expression, called Multiple Sphere Lower Bound (MSLB). This expression can be easily extended in multidimensional signal sets distorted by fading. In order to reveal the accuracy and tightness of the proposed MSLB, numerical and simulations results are provided.

The remainder of the paper is organized as follows. In Section II, the structure and properties of both finite lattices and the system model are described. In Section III, we describe the geometry of the decision regions of the points in an multidimensional finite lattice and we give an expression for the exact performance of such lattices in AWGN channel with Maximum Likelihood (ML) detection. Then we propose a closed-form lower bound of the symbol error probability. The simulation results of various constellations and the analytical bound are compared and discussed in Section IV. Finally, concluding remarks are provided in Section V.

\section{SySTEM MODEL}

\section{A. Finite Lattices}

We consider finite lattice constellations, denoted by $\Lambda^{\prime}$, which are carved from an infinite $N$-dimensional lattice $\Lambda$, for which

$$
\Lambda=\mathbf{M z}, \quad \mathbf{z} \in \mathbb{Z}^{N},
$$


where $\mathbf{M} \in \mathbb{R}^{N \times N}$ is the generator matrix

$$
\mathbf{M}=\left[\begin{array}{llll}
\mathbf{v}_{\mathbf{1}} & \mathbf{v}_{\mathbf{2}} & \ldots & \mathbf{v}_{\mathbf{N}}
\end{array}\right], \quad \mathbf{v}_{\mathbf{i}}=\left[\begin{array}{llll}
v_{i 1} & v_{i 2} & \ldots & v_{i N}
\end{array}\right]^{T},
$$

where $\mathbf{v}_{\mathbf{i}}, i=1, \ldots, N$, are the base vectors of $\Lambda$.

Each of the constellations, $\Lambda^{\prime}$, have $K$ points along the direction of each base vector, forming a parallelotope based on the same vector set. Thus, we assume that these signal sets are constructed by a Pulse Amplitude Modulation (PAM) along the each base vector direction, although this is not the usual consideration of multidimensional signal sets produced by a PAM along every coordinate, since the base vectors are not necessarily orthogonal. Hereafter, a constellation denoted by a $K$-PAM, is a constellation with $K$ points along each base vector direction. That is

$$
\Lambda^{\prime}=\mathbf{M u}, \quad \mathbf{u}=\left[\begin{array}{llll}
u_{1} & u_{2} & \ldots & u_{N}
\end{array}\right]^{T},
$$

and

$$
u_{i} \in\{0,1, \ldots, K-1\} .
$$

The parallelotope consisting of the points

$$
\theta_{1} \mathbf{v}_{\mathbf{1}}+\theta_{2} \mathbf{v}_{\mathbf{2}}+\ldots+\theta_{N} \mathbf{v}_{\mathbf{N}}, \theta_{i}=\{0,1\}
$$

is called fundamental parallelotope of the lattice, a building block which, when repeated, covers the signal space. The volume of the fundamental parallelotope is

$$
\operatorname{vol}(\Lambda)=|\operatorname{det}(\mathbf{M})|
$$

which, for an inner point of a constellation, is equal to the volume of the voronoi cell $\mathcal{V}_{\Lambda}$. As in [13], [14], this volume is normalized to be $|\operatorname{det}(\mathbf{M})|=1$.

\section{B. Communication System Model}

We consider communication in an AWGN channel where the received signal vector is

$$
\mathbf{y}=\mathbf{x}+\mathbf{w},
$$

with $\mathbf{y} \in \mathbb{R}^{N}$ being the received $N$-dimensional real signal vector, $\mathbf{x} \in \mathbb{R}^{N}$ is the transmitted $N$-dimensional real signal vector and $\mathbf{w} \in \mathbb{R}^{N}$ is the $N$-dimensional noise vector whose samples are zero-mean Gaussian independent random variables with variance $\sigma^{2}$. We define the signal-to-noise ratio (SNR) as $\rho=\frac{1}{\sigma^{2}}$.

The probability of receiving $\mathbf{y}$ while transmitting $\mathbf{x}$ is

$$
p(\mathbf{y} \mid \mathbf{x})=\left(2 \pi \sigma^{2}\right)^{-\frac{N}{2}} \exp \left(-\frac{1}{2 \sigma^{2}}\|\mathbf{y}-\mathbf{x}\|^{2}\right)
$$

and Maximum Likelihood (ML) detection is employed at the receiver.

\section{Performance Evaluation via A Combinatorial GEOMETRICAL APPROACH}

The finite lattice constellations under consideration form $N$-dimensional parallelotopes in the $N$-dimensional signal space. Next we present some geometrical aspects of the $N$ dimensional parallelotopes.

\section{A. Geometry of N-dimensional Lattice Parallelotopes}

Every $N$-dimensional parallelotope is constructed by a set of $N$ base vectors, non-orthogonal in the general case, which define the direction of its edges. Specifically, lattice parallelotopes are constructed based on the vectors $\mathbf{v}_{\mathbf{i}}, i=1,2, \ldots, N$, of the infinite lattice $\Lambda$ from which they are carved.

Definition 1: The base vector set is defined as

$$
\mathcal{S}^{N}=\left\{\mathbf{v}_{\mathbf{1}}, \mathbf{v}_{\mathbf{2}}, \ldots, \mathbf{v}_{\mathbf{N}}\right\}
$$

and every subset of $k$ base vectors, $k<N$ as $\mathcal{S}_{m}^{k} \subset \mathcal{S}^{N}$, where $m=1,2, \ldots,\left(\begin{array}{l}N \\ k\end{array}\right)$ is an index enumerating all possible subsets with $k$ out of $N$ vectors.

The vertices, edges, faces etc. of a parallelotope are called facets, laying on corresponding hyperplanes.

Definition 2: A $k$-dimensional facet, $0<k<N$, laying on a hyperplane defined by the vector subset $\mathcal{S}_{m}^{k}$, will be denoted by $P_{m}^{k}$. When $k=N, P^{N}$ denotes the inner space of the $N$-dimensional parallelotope and when $k=0, P^{0}$ denotes a vertice, which we will treat as a 0 -dimensional facet for convenience.

From parallelotope geometry, it holds that

$$
n_{k}=2^{N-k}\left(\begin{array}{c}
N \\
k
\end{array}\right), \quad 0 \leq k \leq N,
$$

where $n_{k}$ is the number of all $P^{k}$ facets on the parallelotope. However, there are only $\left(\begin{array}{l}N \\ k\end{array}\right)$ different types of $P^{k}$ facets on the parallelotope, that is as many as the different base vector subsets. This means that each different $P_{m}^{k}$ appears $2^{n-k}$ times.

Definition 3: We call equivalent- $P_{m}^{k}$, $s$, the multiples of a $P_{m}^{k}$ facet type, appearing on a parallelotope.

For example, a three-dimensional parallelotope, called parallelepiped, consists of three different types of edges $\left(P_{m}^{1}\right.$ facets, $m=1,2,3)$, appearing four times each, and three different types of faces $\left(P_{m}^{2}\right.$ facets, $\left.m=1,2,3\right)$, appearing two times each.

With regard to the signal points of lattice parallelotopes, they can be ranked as inner and outer points. Every outer lattice point lays on a $P_{m}^{k}$ facet with $0 \leq k<N$ and all inner points lay on $P^{N}$. It can be easily deduced that, except from the vertices which are one point each, on a $P_{m}^{k}$ facet the number of points is:

$$
(K-2)^{k}, \quad 0<k \leq N,
$$

where $K$ is the rank of K-PAM.

Definition 4: Points in the same position on equivalent$P_{m}^{k}$ 's are called equivalent points.

For example, Fig. 1 depicts a 4-PAM two-dimensional lattice constellation. In this case, $\mathcal{S}_{1}^{1}=\left\{\mathbf{v}_{\mathbf{1}}\right\}$ and $\mathcal{S}_{2}^{1}=\left\{\mathbf{v}_{\mathbf{2}}\right\}$. We can decern two $P_{1}^{1}$ edges parallel to $\mathbf{v}_{\mathbf{1}}$, two $P_{2}^{1}$ edges parallel to $\mathbf{v}_{\mathbf{2}}$ and four vertices. There are four inner points laying in $P^{n}$, two points on each equivalent $P_{1}^{1}$ and $P_{2}^{1}$ and four vertices in total. Points $A$ and $B$ are equivalent points, since they lay on the same position on the equivalent $P_{2}^{1}$ edges. 


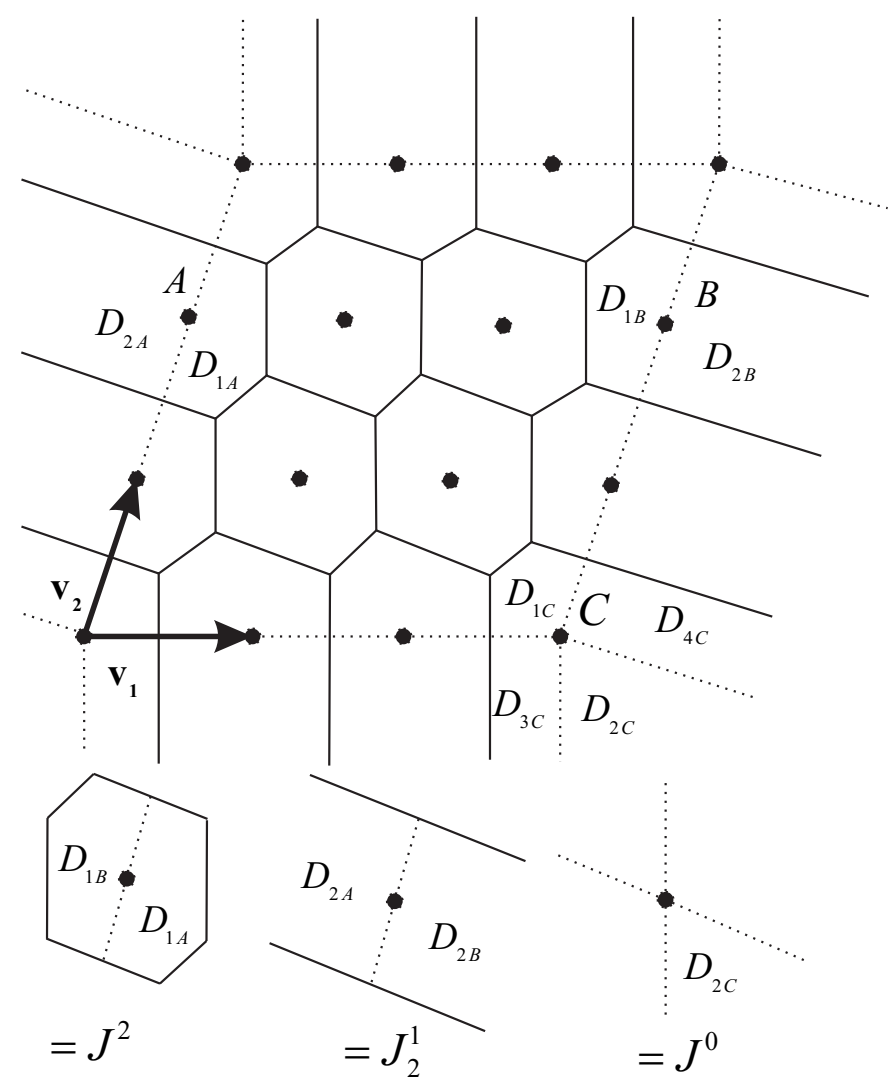

Fig. 1. 2D Lattice and Decision Region Combining

\section{B. Decision Regions Combining}

In order to evaluate the SEP of a lattice constellation, we employ a combinatorial geometrical technique, in order to simplify the evaluation of the integrals on the decision regions of outer points.

The SEP of a constellation is

$$
P(\rho)=1-\mathbb{E}\left[\int_{D} p(\mathbf{z}) \mathrm{d} \mathbf{z}\right],
$$

where $D$ is the region of correct decision of any signal point, $p(\mathbf{z})$ is the $N$-dimensional probability density function of AWGN and $\mathbb{E}[\cdot]$ denotes expectation on all signal points of the constellation, that is $\mathbb{E}\left[\int_{D} p(\mathbf{z}) \mathrm{d} \mathbf{z}\right]=\frac{\mathrm{S}}{K^{N}}$, where $\mathrm{S}$ is the sum of integrals on all decision regions on the constellation.

Remark 1: Every sum of integrals has the property

$$
\int_{D_{1}} p(\mathbf{z}) \mathrm{d} \mathbf{z}+\int_{D_{2}} p(\mathbf{z}) \mathrm{d} \mathbf{z}=\int_{D_{1}+D_{2}} p(\mathbf{z}) \mathrm{d} \mathbf{z},
$$

since $p(\mathbf{z})$ is the same pdf for every transmitted signal point and is spherically symmetrical. In (13), $D_{1}$ and $D_{2}$ must be decision regions which do not overlap when appropriately centered on common coordinate system.

The above property implies that integrals can be divided in partial integrals, and partial integrals can be combined in new, single integrals. For example, in Fig.1 the integrals of the decision regions for points $\mathrm{A}$ and $\mathrm{B}$ are divided in partial integrals on the partial decision regions $D_{1 A}, D_{2 A}, D_{1 B}$ and
$D_{2 B}$. These integrals are combined into two new integrals denoted with $J^{2}$ and $J_{2}^{1}$.

In the case of an $N$-dimensional lattice parallelotope, we divide the decision regions by all the $k$-dimensional hyperplanes, $0<k<N$, which are either facets or vertical to the facets and pass through the vertices of the parallelotope. In the 2D case the above are marked with dashed lines in Fig.1.

Definition 5: The integral $J_{m}^{k}$ is defined as

$$
J_{m}^{k}=\int_{\mathcal{V}\left(\mathcal{S}_{m}^{k}\right)} p(\mathbf{z}) \mathrm{d} \mathbf{z}, \quad 0<k<n,
$$

where $p_{k}(\mathbf{z})$ is a $k$-dimensional zero mean Gaussian distribution, $\mathcal{V}\left(\mathcal{S}_{m}^{k}\right)$ is the voronoi cell of the k-dimensional sublattice defined by the vector subset $\mathcal{S}_{m}^{k}$. When $k=0, J^{0}=1$.

When $k<N$, a $J_{m}^{k}$ integral on combined partial decision regions, is simplified as shown in (14) from $N$-dimensional to $k$-dimensional, computed on the voronoi cell of a $k$ dimensional sublattice. For the downsizing of the dimension of the integrals, a change of variable and a Jacobian transformation as in [11] is used. This downsizing is possible due to the fact that $N-k$ dimensions of the decision region are infinite, something that is not possible in the original decision regions of the signal points.

Remark 2: Partial decision regions of a point which lays on a $P_{m}^{k}$ facet, appear on the adjacent facets of $P_{m}^{k}$ only. When combined with the corresponding integrals of all the equivalent points, laying on the other equivalent $P_{m}^{k}$ facets, they produce a sum of all those integrals $J_{j}^{i}$, for which $\mathcal{S}_{m}^{k} \subseteq \mathcal{S}_{j}^{i}$.

The above remark is an obvious outcome from the following property of the facets of a parallelotope: A $P_{m}^{k}$ facet is adjacent to all those $P_{j}^{i}$ facets, for which $\mathcal{S}_{m}^{k} \subseteq \mathcal{S}_{j}^{i}$. In Fig.1, equivalent points $\mathrm{A}$ and $\mathrm{B}$ lay on the equivalent $P_{2}^{1}$ 's, while $\mathcal{S}_{2}^{1}=\left\{\mathbf{v}_{\mathbf{2}}\right\}$. Thus, the produced integrals are $J_{2}^{1}$ and $J^{2}$, as shown by the combination of $D_{1 A}, D_{1 B}, D_{2 A}$ and $D_{2 B}$, since only $\mathcal{S}_{2}^{1}, \mathcal{S}^{2} \supseteq \mathcal{S}_{2}^{1}$. Moreover, due to supplementary angles appearing between the facets in the parallelotope structure, combined partial regions complete a full $N$-dimensional angle centered at the signal point. For example $2 \pi$ radians for two-dimensional regions, $4 \pi$ steradians for tree-dimensional regions etc. Thus, they add exactly and without overlapping, in the construction of $J_{m}^{k}$ integrals, such as $J^{0}$ in Fig. 1.

\section{Performance Evaluation}

Theorem 1: The SEP of a multidimensional finite lattice constellation is given by

$$
P(\rho)=1-\frac{\sum_{k=0}^{N}(K-1)^{k} \sum_{m=1}^{\left(\begin{array}{c}
N \\
k
\end{array}\right)} J_{m}^{k}}{K^{N}},
$$

where $N$ is the dimension of the lattice, $K$ is the rank of the PAM and $J_{m}^{k}$ 's are defined in (14).

Proof: The proof is given in Appendix A.

The expression in (15) cannot be directly evaluated, since computing analytically the $\mathcal{V}\left(\mathcal{S}_{m}^{k}\right)$ voronoi cells is generally a hard problem [10]. Instead, we propose a lower bound of 
(15), called Multiple Sphere Lower Bound (MSLB), which is constructed by a sum of integrals on multiple spherical integration regions of various dimensions.

Definition 6: We define the integrals

$$
I^{k}=\int_{\mathcal{B}^{k}} p(\mathbf{z}) \mathrm{d} \mathbf{z}, \quad k=1, \ldots, N,
$$

where $\mathcal{B}_{k}$ is a $k$-dimensional sphere, and $I^{0}=1$.

The above integrals can be written as in [13]

$$
I^{k}=\left\{\begin{array}{cc}
1, & k=0 \\
1-\bar{\Gamma}\left(\frac{k}{2}, \frac{R_{k}^{2}}{2} \rho\right), & k=1,2, \ldots, N
\end{array}\right.
$$

where $\bar{\Gamma}(a, x) \triangleq \frac{1}{\Gamma(a)} \int_{x}^{+\infty} t^{a-1} e^{-t} \mathrm{~d} t$ is the normalized upper incomplete Gamma function, $\Gamma(a)$ is the Gamma function defined by [15, Eq. (8.310)] and $R_{k}$ is the radius of $\mathcal{B}^{k}$. The radius $R_{k}$ in AWGN channels, similar to [13] with a slight modification for finite constellations.

Definition 7: The sphere radius is given by

$$
R_{k}^{2}=\left\{\begin{array}{cc}
\frac{1}{\pi} \Gamma\left(\frac{k}{2}+1\right)^{\frac{2}{k}} W^{2}, & k=1,2, \ldots,(N-1) \\
\frac{1}{\pi} \Gamma\left(\frac{k}{2}+1\right)^{\frac{2}{k}}, & k=N
\end{array}\right.
$$

where $W$ is

$$
W=\frac{\left\|\mathbf{v}_{\mathbf{1}}\right\|+\left\|\mathbf{v}_{\mathbf{2}}\right\|+\ldots+\left\|\mathbf{v}_{\mathbf{N}}\right\|}{N},
$$

with $\left\|\mathbf{v}_{\mathbf{i}}\right\|$ being the norm of vector $\mathbf{v}_{\mathbf{i}}$.

Theorem 2: The SEP of a multidimensional finite lattice constellation is lower bounded by

$$
P_{m s l b}(\rho)=1-\frac{\sum_{k=0}^{N}(K-1)^{k}\left(\begin{array}{c}
N \\
k
\end{array}\right) I^{k}}{K^{N}}
$$

where $P_{m s l b}(\rho)$ is called Multiple Sphere Lower Bound (MSLB), $\rho=\frac{1}{\sigma^{2}}, N$ is the dimension of the lattice, $K$ is the rank of $K$-PAM and $I^{k}$ 's are the spherical integrals given in closed form by (17),(18) and (19).

Proof: The proof is given in Appendix B.

\section{Numerical Results \& Discussion}

In this section we illustrate the accuracy and tightness of the proposed MSLB, in comparison with the exact SEP of various lattice constellations in AWGN channels, obtained via simulations. The lattice constellations most commonly used in practical cases, due to the easy gray coded bit labeling, are those carved from $\mathbb{Z}^{N}$ lattices. In the following, apart from $\mathbb{Z}^{N}$ lattices, sphere packing lattice constellations such as the $\mathbb{A}^{2}$ are also illustrated. These constellations achieve better energy efficiency but they cannot be labeled with a gray code. In all $\mathbb{Z}^{N}$ lattices, the normalization of the volume of the voronoi cell yields $W=1$, while for the $\mathbb{A}^{2}$ lattice, it yields $W=$ $\sqrt{\frac{2}{\sqrt{3}}}$.

Fig. 2 compares the MSLB with the SEP of $\mathbb{Z}^{N}$ 4-PAM constellations for different values of the lattice dimension.

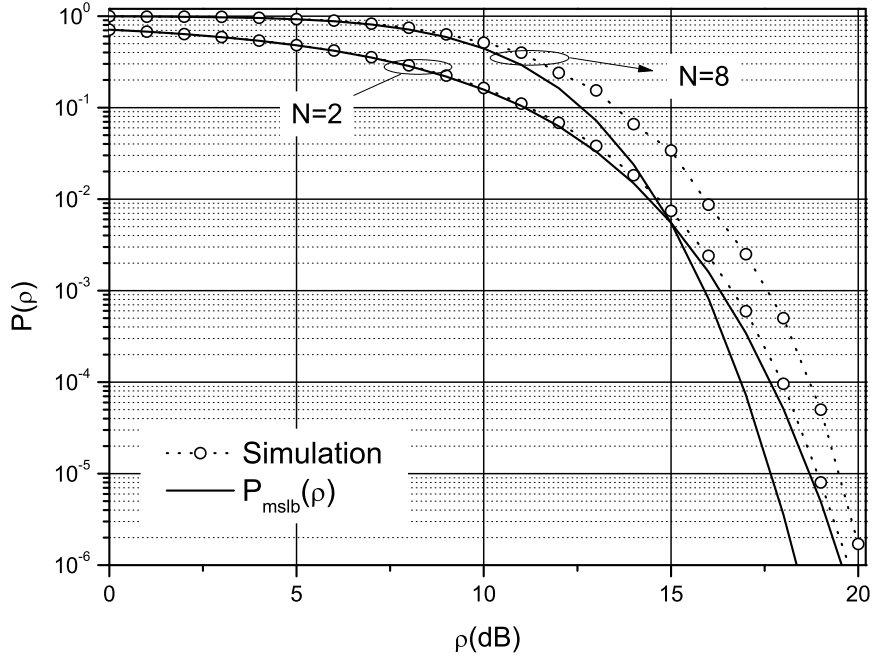

Fig. 2. Symbol Error Probability, $P(\rho)$, and MSLB for $\mathbb{Z}^{N} 4-P A M$ constellations with $N=2,8$.

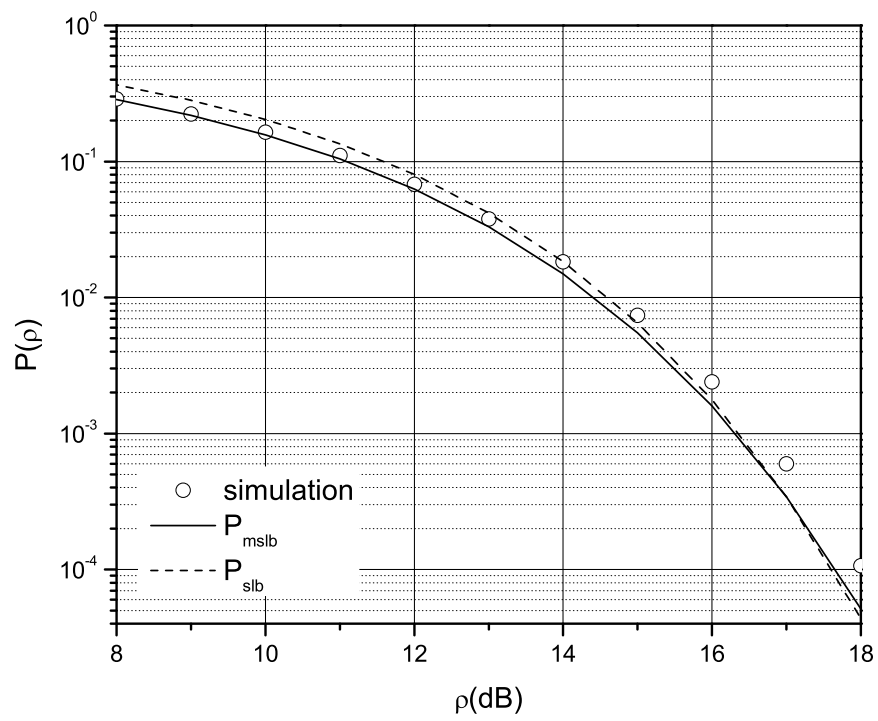

Fig. 3. Symbol Error Probability, $P(\rho)$, MSLB and SLB for a $\mathbb{Z}^{2} 4-P A M$ constellation $(N=2)$.

Specifically, MSLB is plotted in conjunction with the simulation results for the SEP of the $\mathbb{Z}^{N}$ 4-PAM constellations, for $N=2,8$. It is clearly illustrated that the MSLB acts as a lower bound, regardless of the dimension of the constellation. Furthermore, as the dimension decreases, the bound becomes very tight. This was expected since the ratio inner/outer points on the constellation increases rapidly and the approximation of the Voronoi cells of the inner points is better than that of the outer points in the MSLB.

Fig. 3 is a closeup on the SEP of $\mathbb{Z}^{N}$ 4-PAM constellations plotted together with the MSLB and SLB for the corresponding infinite lattice. It is evident that the conventional SLB is not a lower bound for a finite constellation. Moreover, the MSLB is tighter than the SLB with respect to the simulated constellation performance. 


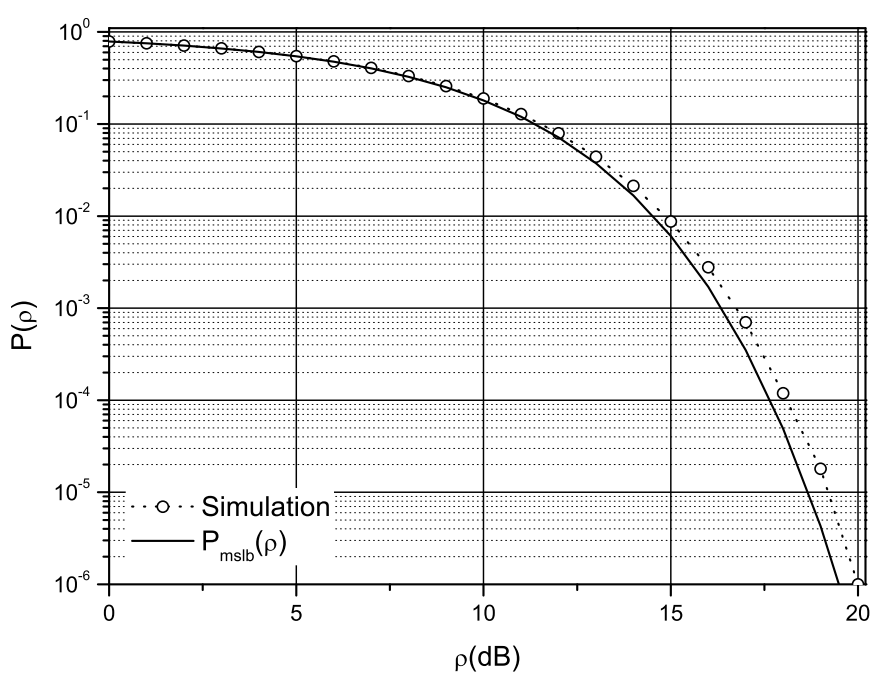

Fig. 4. Symbol Error Probability, $P(\rho)$, and MSLB for a $\mathbb{Z}^{2} 8-P A M$ constellation $(N=2)$.

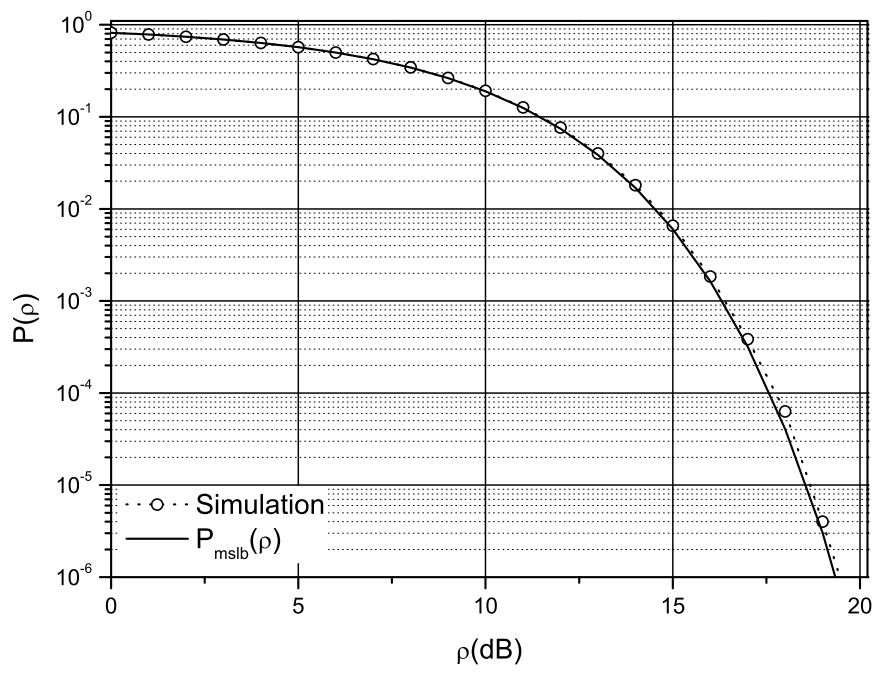

Fig. 5. Symbol Error Probability, $P(\rho)$, and MSLB for an $\mathbb{A}^{2} 16-P A M$ constellation $(N=2)$.

Fig. 4 depicts the MSLB along with the SEP of the $\mathbb{Z}^{2}$ 8-PAM constellation. It is clearly illustrated that the MSLB bounds the performance of the lattice and it is still tight, as the rank $K$ of the $K$-PAM increases. Consequently, the expression of the MSLB acts as a lower bound of the SEP, regardless of the rank of the $K$-PAM of the constellations.

Finally, Fig. 5 depicts an $\mathbb{A}^{2} 16-\mathrm{PAM}$ constellation, along with the MSLB. Once again it is clear that MSLB acts as a lower bound. In fact, it is observed that in this case the proposed bound is more tight than any other lattice compared. This can be explained by taking into account that the Voronoi cells of sphere packing lattices are regular polytopes, which are better approximated by the spheres of MSLB.

\section{CONCLUSiOns}

We have studied the error performance of finite lattice constellations via a combinatorial geometrical approach. First we presented an analytical expression for the exact SEP of these signal sets. This was used to provide a novel closedform analytical bound, called Multiple Sphere Lower Bound (MSLB), which lower-bounds the SEP of multidimensional finite lattice constellations. The tightness of MSLB has been illustrated in comparison with the simulated SEP of various constellations. It was demonstrated that the derived MSLB can be efficiently used as a benchmark for the error performance of multidimensional finite lattice constellations in AWGN channels, regardless of the lattice structure, dimension or rank of the constellation. Furthermore, the presented approach can be extended to the analysis of multidimensional signal sets distorted by fading; thus, illustrating the diversity gains offered by signal space diversity ${ }^{1}$.

\section{APPENDIX A}

\section{ProOF OF THEOREM 1}

Due to Remark 2, the sum of partial regions of equivalent points, laying on all equivalent $P_{m}^{k}$,s, for specific $k$ and $m$, yields the sum of integrals,

$$
\begin{array}{ll}
\sum_{i=k}^{N} \sum_{j: \mathcal{S}_{m}^{k} \subseteq \mathcal{S}_{j}^{i}} J_{j}^{i}, & 0<k \leq N \\
\sum_{i=0}^{N} \sum_{j=1}^{N} J_{j}^{i}, & k=0 .
\end{array}
$$

From (11) and (21), all points laying on $P_{m}^{k}$ facets for specific $k$ and $m$, provide the sum of integrals,

$$
\begin{array}{lll}
(K-2)^{k} \sum_{i=k}^{N} \sum_{j: \mathcal{S}_{m}^{k} \subseteq \mathcal{S}_{j}^{i}} J_{j}^{i}, & 0<k<N, \\
\sum_{i=0}^{N} \sum_{j=1}^{\left(\begin{array}{c}
N \\
i
\end{array}\right)} J_{j}^{i}, & k=0 .
\end{array}
$$

Adding the above sums for all values of $m$ and $k$, we have

$$
\mathrm{S}=\sum_{k=1}^{N}(K-2)^{k} \sum_{m=1}^{\left(\begin{array}{c}
N \\
k
\end{array}\right)} \sum_{i=k}^{N} \sum_{j: \mathcal{S}_{m}^{k} \subseteq \mathcal{S}_{j}^{i}} J_{j}^{i}+\sum_{i=0}^{N} \sum_{j=1}^{\left(\begin{array}{c}
N \\
i
\end{array}\right)} J_{j}^{i}
$$

which can be equivalently written as

$$
\mathrm{S}=\sum_{k=0}^{N} \sum_{i=0}^{k}\left(\begin{array}{c}
k \\
i
\end{array}\right)(K-2)^{i} \sum_{m=1}^{\left(\begin{array}{c}
N \\
k
\end{array}\right)} J_{m}^{k}
$$

or using the bionomial theorem [15, Eq. (1.111)] yields

$$
\mathrm{S}=\sum_{k=0}^{N}(K-1)^{k} \sum_{m=1}^{\left(\begin{array}{c}
N \\
k
\end{array}\right)} J_{m}^{k}
$$

Using (25), (12) yields (15) and this concludes the proof.

\footnotetext{
${ }^{1}$ Due to space limitations, the extension of the MLSB to fading channels will be included in the journal version of the paper.
} 


\section{APPENDIX B \\ PROOF OF THEOREM 2}

For the volume of $\mathcal{V}\left(\mathcal{S}_{m}^{k}\right)$ in (14), since it is the voronoi cell of a sublattice built by the vector subset $\mathcal{S}_{m}^{k}$, it holds that

$$
\operatorname{vol}\left(\mathcal{V}\left(\mathcal{S}_{m}^{k}\right)\right) \leq \prod_{i: \mathbf{v}_{i} \in \mathcal{S}_{m}^{k}}\left\|\mathbf{v}_{i}\right\|
$$

where $\left\|\mathbf{v}_{i}\right\|$ is the norm of $\mathbf{v}_{i}$, with the equality holding only when the vectors of $\mathcal{S}_{m}^{k}$ are orthogonal, while $\operatorname{vol}(\cdot)$ is the volume of a region.

Furthermore, since the probability density function of AWGN is spherically symmetrical, it holds that

$$
\int_{D} p(\mathbf{z}) \mathrm{d}(\mathbf{z}) \leq \int_{\mathcal{B}_{D}} p(\mathbf{z}) \mathrm{d} \mathbf{z}
$$

when $\operatorname{vol}(D)=\operatorname{vol}\left(\mathcal{B}_{D}\right)$, as in [13]. In (27) $D$ is a random $k$ dimensional region of integration and $\mathcal{B}_{D}$ is a $k$-dimensional sphere. In general, (27) is also valid when $\operatorname{vol}(D) \leq \operatorname{vol}\left(\mathcal{B}_{D}\right)$.

Since the AWGN pdf is a decreasing function with respect to the distance from the center, it holds that for some $L$,

$$
\sum_{i=1}^{L} \int_{D_{i}} p(\mathbf{z}) \mathrm{d} \mathbf{z} \leq L \int_{\overline{\mathcal{B}}} p(\mathbf{z}) \mathrm{d} \mathbf{z}
$$

as long as

$$
\sum_{i=1}^{L} \operatorname{vol}\left(D_{i}\right) \leq L \operatorname{vol}(\overline{\mathcal{B}})
$$

where $L \in \mathbb{Z}, D_{i}$ 's are random different $k$-dimensional volumes of integration and $\overline{\mathcal{B}}$ is a $k$-dimensional sphere.

For some $k$ in (25), we substitute $J_{m}^{k}$ for every $m$ with an integral $I^{k}$ as in (16),(17). We set $\operatorname{vol}\left(\mathcal{B}^{k}\right)=W^{k}$, where $W$ is as in (19), and the radius of the sphere is calculated by (18). From (26) it holds that

$$
\sum_{m=1}^{\left(\begin{array}{c}
N \\
k
\end{array}\right)} \operatorname{vol}\left(\mathcal{V}\left(\mathcal{S}_{m}^{k}\right)\right) \leq \sum_{m=1} \prod_{i: \mathbf{v}_{i} \in \mathcal{S}_{m}^{k}}\left\|\mathbf{v}_{i}\right\|
$$

which can be written as

$$
\sum_{m=1}^{\left(\begin{array}{c}
N \\
k
\end{array}\right)} \operatorname{vol}\left(\mathcal{V}\left(\mathcal{S}_{m}^{k}\right)\right) \leq \sum_{\substack{b_{1}+b_{2}+\ldots+b_{N}=k \\
b_{1}, b_{2}, \ldots, b_{N} \in\{0,1\}}}\left\|\mathbf{v}_{1}\right\|^{b_{1}}\left\|\mathbf{v}_{2}\right\|^{b_{2}} \cdots\left\|\mathbf{v}_{N}\right\|^{b_{N}}
$$

Using Maclaurin's Inequality [16, p.52], for $a_{1}, a_{2}, \ldots, a_{N} \in \mathbb{R}$ and any $0<k<N$ holds,

$$
\varrho_{N}^{\frac{1}{N}} \leq \varrho_{k}^{\frac{1}{k}} \leq \varrho_{1}
$$

where

$$
\varrho_{k}=\frac{\sum_{\substack{b_{1}+b_{2}+\ldots+b_{N}=k \\
b_{1}, b_{2}, \ldots, b_{N} \in\{0,1\}}} a_{1}^{b_{1}} a_{2}^{b_{2}} \cdots a_{N}^{b_{N}}}{\left(\begin{array}{c}
N \\
k
\end{array}\right)}
$$

If we set $a_{i}=\left\|\mathbf{v}_{i}\right\|, i=1,2, \ldots, N$, then $\varrho_{1}=W$ and from (32) and (33)

$$
\sum_{\substack{b_{1}+b_{2}+\ldots+b_{N}=k \\
b_{1}, b_{2}, \ldots, b_{N} \in\{0,1\}}}\left\|\mathbf{v}_{1}\right\|^{b_{1}}\left\|\mathbf{v}_{2}\right\|^{b_{2}} \cdots\left\|\mathbf{v}_{N}\right\|^{b_{N}} \leq\left(\begin{array}{c}
N \\
k
\end{array}\right) W^{k}
$$

From (31), (34) and since $\operatorname{vol}\left(\mathcal{B}^{k}\right)=W^{k}$, we have

$$
\sum_{m=1}^{\left(\begin{array}{c}
N \\
k
\end{array}\right)} \operatorname{vol}\left(\mathcal{V}\left(\mathcal{S}_{m}^{k}\right)\right) \leq\left(\begin{array}{l}
N \\
k
\end{array}\right) \operatorname{vol}\left(\mathcal{B}^{k}\right)
$$

According to (28) and (29), (35) leads to

$$
\sum_{m=1}^{\left(\begin{array}{c}
N \\
k
\end{array}\right)} J_{m}^{k} \leq\left(\begin{array}{c}
N \\
k
\end{array}\right) I^{k}
$$

Using (15), (20) and (36), it holds that

$$
P_{m s l b}(\rho) \leq P(\rho)
$$

and this concludes the proof.

\section{REFERENCES}

[1] J. Boutros and E. Viterbo, "Signal Space Diversity: A power- and bandwidth-efficient diversity technique for the Rayleigh fading channel", IEEE Trans. Inf. Theory, vol. 44, no. 4, p. 1453-1467, Jul. 1998.

[2] J. Boutros, E. Viterbo, C. Rastello, and J. C. Belfiore, "Good lattice constellations for both Rayleigh fading and Gaussian channels", IEEE Trans. Inf. Theory, vol. 42, no. 2 pp. 502-518, Mar. 1996.

[3] X. Giraud, E. Boutillon, and J. C. Belfiore, "Algebraic tools to build modulation schemes for fading channels", IEEE Trans. Inf. Theory, vol. 43, no. 3, pp. 938-952, May 1997.

[4] E. Bayer-Fluckiger, F. Oggier, and E. Viterbo, "New algebraic constructions of rotated Zn-lattice constellations for the Rayleigh fading channel," IEEE Trans. Inf. Theory, vol. 50, no. 4, pp. 702-714, Apr. 2004.

[5] F. Oggier and E. Viterbo, Algebraic Number Theory And Code Design For Rayleigh Fading Channels, Foundations and Trends in Communications and Information Theory. Now Publishers Inc, 2004.

[6] J.-C. Belfiore and E. Viterbo, "Approximating the error probability for the independent Rayleigh fading channel", in Proc. 2005 International Symposium on Information Theory, Adelaide, Australia, Sept. 2005.

[7] G. Taricco and E. Viterbo, "Performance of high-diversity multidimensional constellations", IEEE Trans. Inf. Theory, vol. 44, no. 4, pp. 15391543, Jul 1998.

[8] J. Kim, W. Lee, J.-K. Kim, and I. Lee, "On the symbol error rates for signal space diversity schemes over a Rician fading channel", IEEE Trans. Commun., vol. 57, no. 8, pp. 2204-2209, Aug. 2009.

[9] C. E. Shannon, "Probability of error for optimal codes in a Gaussian channel", The Bell Syst. Techn. J., vol. 38, no. 3, pp. 279-324, May 1959

[10] E. Viterbo and E. Biglieri, "Computing the voronoi cell of a lattice: the diamond-cutting algorithm," IEEE Trans. Inf. Theory, vol. 42, no. 1, p. 161-171, Jan. 1996.

[11] V. Tarokh, A. Vardy, and K. Zeger, "Universal bound on the performance of lattice codes," IEEE Trans. Inf. Theory, vol. 45, no. 2, pp. 670-681, Mar. 1999.

[12] S. Vialle and J. Boutros, "Performance of optimal codes on Gaussian and Rayleigh fading channels: a geometrical approach", in Proc. 37th Allerton Conf. on Commun., Control and Comput., Monticello, IL, Sept. 1999.

[13] A. G. Fàbregas and E. Viterbo, "Sphere lower bound for rotated lattice constellations in fading channels", IEEE Trans. Wireless Commun., vol. 7, no. 3, p. 825-830, Mar. 2008

[14] J. H. Conway and N. J. A. Sloane, Sphere Packings, Lattices and Groups, 3rd ed. Springer, 1999.

[15] I. S. Gradshteyn and I. M. Ryzhik, Table of Integrals, Series, and Products, New York, Academic Press, 7th edition, 2007.

[16] G.H. Hardy, J.E. Littlewood, and G. Polya, Inequalities, 2nd ed. Cambridge, UK, Cambridge Univ. Press, 1952. 